\title{
Viabilidade e vigor de sementes de paineira após armazenamento, condicionamento e estresses salino e térmico
}

\author{
Sonia Cristina Juliano Gualtieri de Andrade Perez ${ }^{(1)}$ e Manoela Meyrsieck Jardim ${ }^{(1)}$
}

\author{
(1)Universidade Federal de São Carlos, Dep. de Botânica, Caixa Postal 676, CEP 13565-905 São Carlos, SP. E-mail: dscp@power.ufscar.br, \\ manujardim@hotmail.com
}

\begin{abstract}
Resumo - O objetivo deste trabalho foi avaliar o efeito do condicionamento e dos estresses salino e térmico sobre o vigor e a viabilidade de sementes de paineira (Chorisia speciosa St.-Hil) armazenadas em geladeira, em embalagens impermeáveis, durante três anos. Sementes com e sem punção do tegumento foram condicionadas em água destilada e em soluções de $\mathrm{KNO}_{3}$ a 0,1 M e 0,2 M, durante 24 horas, a $27^{\circ} \mathrm{C}$. O estresse salino foi simulado com soluções de $\mathrm{NaCl}$ em potenciais osmóticos variando de 0,0 a $-1,2 \mathrm{MPa}$, a $27^{\circ} \mathrm{C}$. O estresse térmico de diferentes intensidades foi aplicado em sementes secas, que germinaram a $27^{\circ} \mathrm{C}$. Houve diminuição da viabilidade e do vigor das sementes com o aumento da intensidade dos estresses salino e térmico. Nas sementes condicionadas e intactas, o limite máximo de tolerância à salinidade não foi reduzido, porém houve diminuição da tolerância ao estresse térmico, em sementes condicionadas com ou sem punção. O teste de condutividade elétrica indicou as sementes condicionadas em água e $\mathrm{KNO}_{3} 0,1 \mathrm{M}$ como as de maior vigor. Sementes condicionadas em água apresentaram maior velocidade de germinação sob estresses salino e térmico. O condicionamento substituiu a punção do tegumento, que não é recomendada para sementes armazenadas.
\end{abstract}

Termos para indexação: Bombacaceae, Chorisia speciosa, osmocondicionamento, salinidade, temperatura, espécie florestal tropical.

\section{Viability and vigor in folk silk tree seeds after storage, conditioning and submission to salt and thermal stress}

\begin{abstract}
The aim of this work was to evaluate the effects of priming, salt and thermal stress on viability and vigor of folk silk tree seeds (Chorisia speciosa St.-Hil) after storage in hermetic containers at $5^{\circ} \mathrm{C}$. Intact and with coat punction seeds were primed in distilled water and $\mathrm{KNO}_{3}$ solutions $(0.1 \mathrm{M}$ and $0.2 \mathrm{M})$ during 24 hours at $27^{\circ} \mathrm{C}$. Salt stress was simulated with $\mathrm{NaCl}$ solutions at different osmotic potential, ranging from $0.0 \mathrm{MPa}$ until $-1.2 \mathrm{MPa}$, at $27^{\circ} \mathrm{C}$. Different intensities of thermal stress were applied on dried seeds; germination tests were carried out at $27^{\circ} \mathrm{C}$. As the intensity of salt and thermal stress increased, a reduction on seed vigor and viability was recorded in intact or punctured seeds. The salt tolerance limit showed before the storage was not reduced in intact seeds but there was an increase at thermal sensibility on intact or punctured seeds. The highest rates and germination percentage are recorded in seeds primed in water, submitted to salt and thermal stress. The conductivity test indicated the seeds primed in water or $\mathrm{KNO}_{3} 0.1 \mathrm{M}$ as the most vigorous. It is desirable to use priming to substitute the coat punction, after seed storage.

Index terms: Bombacaceae, Chorisia speciosa, osmotic conditioning, salinity, temperature, wood tropical species.
\end{abstract}

\section{Introdução}

Tratamentos pós-colheita têm sido usados com o objetivo de aumentar a velocidade de germinação e o desempenho das sementes e plântulas. Entre os métodos empregados para aumentar a expressão do vigor das sementes, um dos mais utilizados é o osmocondicionamento, que consiste na incubação das sementes em soluções com agentes osmóticos, durante um período específico e temperatura predeterminada (Taylor et al., 1998).

Os resultados positivos do condicionamento osmótico, sobretudo para culturas agrícolas, reafirmam seu potencial para melhorar o processo germinativo e o vigor das sementes. Porém, existem poucas informações a respeito do condicionamento osmótico em sementes de espécies florestais nativas. O condicionamento, associado às condições ideais de armazenamento, pode me- 
lhorar o aproveitamento das sementes de espécies florestais, cuja produção de frutos nem sempre é regular e a coleta de sementes é trabalhosa e onerosa (Carpi et al., 1996).

Com a utilização de técnicas especiais de conservação ou recuperação do potencial germinativo, muitas das espécies que produzem sementes de baixa longevidade, em condições naturais, têm sua longevidade prolongada, como é o caso de Cedrela angustifolia (Pinã-Rodrigues \& Jesus, 1992) e Cedrela fissilis (Carpi et al., 1996).

Em solos de regiões tropicais, a água subterrânea é a principal fonte de suprimento hídrico para as espécies florestais e está sujeita à salinização freqüente, causada pela alta força evaporativa do ar, que leva à saída de água da superfície do solo, tornando-a mais salinizada do que as camadas mais profundas. Sais contendo sódio, cálcio e magnésio são os mais comuns e os elevados níveis de salinização afetam o potencial hídrico do solo, pH e a atividade microbiana. Em geral, as sementes estão em ambiente mais salinizado do que as plântulas já estabelecidas, cujas raízes podem usar a porção menos salinizada do perfil do solo (Agboola, 1998).

Em sementes condicionadas, vários trabalhos registraram um aumento de tolerância a diferentes tipos de estresse. Por exemplo, foi observada germinação mais rápida em temperaturas mais baixas que a ótima por Jeller \& Perez (2003), maior resistência ao estresse hídrico (Wanli et al., 2001; Fanti \& Perez, 2003) e ao estresse salino (Perez \& Negreiros, 2001), aumento significativo na produtividade (Muhyaddin \& Wiebe, 1989) e aumento da matéria seca das plântulas (Suñe et al., 2002).

Neste estudo foram utilizadas sementes de Chorisia speciosa St.-Hil (paineira), espécie arbórea, caducifólia, pertencente à família Bombacaceae. A espécie é encontrada em florestas mesófilas semidecíduas dos estados de São Paulo, Minas Gerais, Goiás, Mato Grosso do Sul, Paraná, Santa Catarina, Rio Grande do Sul, como também no nordeste da Argentina e centro-oeste do Paraguai. A madeira é empregada na confecção de canoas, cochos, caixotaria, forros de móveis e no fabrico de pasta celulósica. Por seu grande efeito ornamental, porte avantajado e beleza das flores, a espécie é freqüentemente cultivada em praças, jardins e avenidas. É recomendada para plantios mistos em áreas degradadas de preservação, reconstituição de matas, principalmente em áreas de solos úmidos (Carvalho, 1994).
O objetivo deste trabalho foi avaliar o efeito do condicionamento e de estresses salino e térmico na viabilidade e vigor de sementes de paineira armazenadas, em geladeira, em embalagens impermeáveis, durante três anos.

\section{Material e Métodos}

O trabalho foi conduzido no Dep. de Botânica da Universidade Federal de São Carlos. Foram utilizadas sementes selecionadas de paineira fornecidas pelo Instituto de Pesquisa e Estudos Florestais de Piracicaba da Esalq/USP, provenientes da cidade de Maristela, SP, com $11 \%$ de umidade, armazenadas durante três anos, em embalagens impermeáveis, em geladeira a $5^{\circ} \mathrm{C}$. Realizou-se uma triagem manual dessas sementes para garantir uniformidade de tamanho, coloração e estado de conservação.

No condicionamento, metade das sementes previamente pesadas permaneceu intacta, e a outra metade recebeu uma punção no tegumento (Fanti, 2001). A seguir, as sementes foram distribuídas em bandejas de alumínio $(28 \times 38 \mathrm{~cm})$, forradas com duas folhas de papel-filtro umedecidas com $80 \mathrm{~mL}$ das soluções de $\mathrm{KNO}_{3}$ 0,1 M e 0,2 M e água destilada. As bandejas foram introduzidas em um saco de plástico transparente para evitar a evaporação da solução, e o condicionamento foi realizado durante 24 horas a $27^{\circ} \mathrm{C}$. Após este tratamento, as sementes foram lavadas, submetidas à secagem, sob $27^{\circ} \mathrm{C}$ até atingirem o peso inicial, apresentado antes do condicionamento. Assim, as sementes sem condicionamento (controle) e as sementes condicionadas em água destilada e em soluções de $\mathrm{KNO}_{3}$, que receberam ou não a punção do tegumento, formaram os diferentes grupos de sementes que foram submetidas ao estresse salino e térmico e ao teste de condutividade.

$\mathrm{O}$ estresse salino foi simulado com $\mathrm{NaCl}$ nos potenciais osmóticos variando desde 0,0 MPa até -1,2 MPa, em intervalos de -0,2 MPa, na temperatura ótima constante de $27^{\circ} \mathrm{C}$ (Fanti, 2001). As soluções de $\mathrm{KNO}_{3}$ foram preparadas de acordo com a equação de Vant' Hoff (Hillel, 1971, citado por Salisbury \& Ross, 1994).

As sementes permaneceram durante 24 , 48 e 72 horas sob as temperaturas de $50^{\circ} \mathrm{C}, 60^{\circ} \mathrm{C}$ e $70^{\circ} \mathrm{C}$ e, em seguida, foram colocadas para germinar sob temperatura ótima de $27^{\circ} \mathrm{C}$, de acordo com Fanti (2001).

As leituras de germinação foram realizadas a cada 24 horas, considerando-se como sementes germinadas as que apresentavam emissão de raiz primária maior ou 
igual a 2 mm e curvatura geotrópica positiva (Labouriau, 1983). O experimento foi finalizado quando todas as sementes haviam germinado ou quando as remanescentes apresentavam-se deterioradas.

Os valores de porcentagem e velocidade de germinação foram calculados de acordo com fórmulas citadas em Labouriau (1983). O delineamento estatístico utilizado foi inteiramente casualizado, com quatro repetições de 25 sementes para cada estudo proposto (estresses salino, térmico e teste de condutividade). $\mathrm{Na}$ análise estatística dos dados, foi empregado o teste de Bartllet para verificar a presença de homocedasticidade e, em seguida, realizada a análise de variância (duas entradas) e teste de Tukey-Kramer para contraste das médias. Os valores médios de porcentagem foram transformados para arc sen $(\mathrm{P} / 100)^{0,5}$ e de velocidade de germinação $(V)$ foram transformados em log $(V+1)$, em alguns casos, com o uso do programa estatístico InSTAT (GraphPad, 1999). Para o estresse salino, foi utilizado o esquema fatorial de $4 \times 7$ (soluções de condicionamento e potenciais osmóticos do meio germinativo) e para o estresse térmico, o esquema fatorial de $4 \times 3 \times 3$ (soluções de condicionamento, temperaturas e tempo de exposição). Na presença de interações significativas entre os fatores, foram feitos os desdobramentos dos dois fatores envolvidos em cada uma das análises (Zar, 1999).

\section{Resultados e Discussão}

Antes de serem armazenadas, as sementes apresentaram 96\% de germinação em 3,84 dias e, após três anos de armazenamento, houve uma redução de $26 \%$ na viabilidade das sementes e um atraso no processo de germinação. As sementes de paineira recém-colhidas, e que receberam a punção do tegumento, apresentaram um limite máximo de tolerância ao sal entre-1,0 MPa e-1,2 MPa(Fanti, 2001). Após três anos de armazenamento, as sementes que receberam a punção do tegumento apresentaramse mais sensíveis ao estresse salino, com um limite de tolerância entre -0,6 MPa e -0,8 MPa, e menores valores de velocidade de germinação. As sementes mantidas intactas exibiram o mesmo limite de tolerância apresentado antes do armazenamento (Tabela 1).

Em sementes que receberam a punção do tegumento, constatou-se uma redução significativa da porcentagem de germinação a partir de -0,2 MPa, havendo interação entre potencial osmótico do meio e solução de condicionamento. $\mathrm{O}$ uso da pré-embebição de sementes em água aumentou a porcentagem de germinação, em relação ao controle e às sementes condicionadas com $\mathrm{KNO}_{3}$. Com relação à velocidade de germinação, observou-se redução dos valores a partir de -0,2 MPa, independentemente das soluções de condicionamento utilizadas. Os maiores valores de velocidade foram obtidos em sementes condicionadas em água, mas não se verificou interação entre os fatores potencial osmótico do meio e soluções de condicionamento (Tabela 1).

As sementes intactas e condicionadas, que permaneceram em solução salina, apresentaram redução da viabilidade a partir de -0,4 MPa. As sementes que foram condicionadas em água ou $\mathrm{KNO}_{3}$ apresentaram maiores valores de porcentagem de germinação. A partir de -0,8 MPa, o condicionamento não foi efetivo em promover um aumento da porcentagem de germinação. Com relação à velocidade, uma redução significativa dos valores foi registrada a partir de -0,6 MPa e o condicionamento em água aumentou a velocidade de germinação em relação aos outros tipos de solução (Tabela 1).

$\mathrm{O}$ sal $\mathrm{KNO}_{3}$ a $0,1 \mathrm{M}$ aumentou a velocidade e porcentagem de germinação de sementes de paineira, sem armazenamento, sob estresse hídrico (Fanti \& Perez, 2003). Porém, existe a possibilidade do uso de outras soluções como as de manitol, polietilenoglicol, e sais inorgânicos como o $\mathrm{NaCl}, \mathrm{MgSO}_{4}$ e $\mathrm{KNO}_{3}$, uma vez que a eficiência destas soluções é variável entre as espécies (Vasquez, 1995).

Por exemplo, soluções de PEG 6000 foram mais eficientes que as de $\mathrm{KNO}_{3}$ no condicionamento de sementes de Cassia excelsa e produziram aumento na porcentagem de germinação em temperaturas sub e supraótimas e sob estresse salino (Jeller \& Perez, 2003).

Braccini et al. (1999) observaram efeitos prejudiciais às sementes, com o uso de condicionamento em água, devido à rápida embebição, que pode reduzir a integridade das membranas, compostas de uma dupla camada de fosfolipídeos. Essa composição é dependente da água para manter a sua estrutura e, assim, as membranas da semente seca podem estar no estado gel, não funcionando como uma barreira eficiente à lixiviação de compostos celulares. Assim, se a embebição for muito rápida, não haverá tempo para que as membranas voltem ao estado cristalino líquido, ocasionando danos celulares e lixiviação de solutos (Castro \& Hilhorst, 2004).

Os resultados são similares àqueles em que o aumento da salinidade diminui a porcentagem e a velocidade de germinação das sementes. Porém, o efeito das soluções salinas na germinação é complexo. As discrepâncias são 
numerosas, assim como os limites entre efeitos tóxicos e osmóticos, predominantes de um sal, não são definidos. Em alguns casos, pode haver efeito promotor via destruição da testa das sementes, facilitando a embebição (Agboola, 1998).

O condicionamento pode reverter alterações prejudiciais nas membranas celulares como a inativação de enzimas, a inibição da síntese protéica, ocasionadas por elevadas concentrações de $\mathrm{Na}^{+}$que deslocam $\mathrm{Ca}^{2+}$ da membrana, afetando a permeabilidade dessas, e este fato é detectado pela saída de $\mathrm{K}^{+}$das células (Taiz \& Zeiger, 2004).

A técnica do condicionamento permite a hidrólise de reservas armazenadas e a síntese de materiais necessários à germinação, fazendo com que ocorra uma germinação mais rápida e homogênea, diminuindo o tempo de exposição às condições desfavoráveis, como deficiência hídrica, salinidade, estresse térmico. Assim, o condicionamento promove uma emergência mais rápida e uniforme das plântulas, propiciando maior vigor sob condições adversas (Warren \& Benett, 1997).

As sementes de paineira, sem armazenamento, apresentaram tolerância ao estresse térmico aplicado nas temperaturas de 50,60 e $70^{\circ} \mathrm{C}$ durante 24 , 48 e 72 horas (Tabela 2). A porcentagem de germinação foi reduzida para $50 \%$ quando as sementes foram expostas a $70^{\circ} \mathrm{C}$ durante 72 horas (Fanti, 2001). Após três anos de armazenamento em geladeira, foi registrado aumento da sensibilidade ao estresse térmico nas sementes de paineira (Tabela 2).

Além disso, houve inibição total da germinação a $70^{\circ} \mathrm{C}$, em sementes que receberam ou não a punção do tegumento, evidenciando que a tolerância ao estresse depende do genótipo, associado à condição fisiológica da semente.

Não se observou interação entre os fatores soluções de condicionamento e tempo de exposição ao estresse, tanto para velocidade quanto para porcentagem de germinação (Tabela 2). Independentemente da temperatu-

Tabela 1. Germinação [arc sen $\left.(\mathrm{P} / 100)^{0,5}\right]$ e velocidade de germinação [log (v + 1)] de sementes de Chorisia speciosa, armazenadas durante três anos, a $5^{\circ} \mathrm{C}$, submetidas ou não ao condicionamento com ou sem punção do tegumento, e, em seguida, expostas ao estresse salino com soluções de $\mathrm{NaCl}^{(1)}$.

\begin{tabular}{|c|c|c|c|c|c|c|c|}
\hline \multirow[t]{2}{*}{ Condicionamento } & \multicolumn{6}{|c|}{ Potencial osmótico do meio germinativo com $\mathrm{NaCl}(\mathrm{MPa})$} & \multirow{2}{*}{ Média } \\
\hline & 0,0 & $-0,2$ & $-0,4$ & $-0,6$ & $-0,8$ & $-1,0$ & \\
\hline & \multicolumn{7}{|c|}{ Germinação de sementes com punção do tegumento } \\
\hline Controle & $58,30 \mathrm{Aa}$ & $58,30 \mathrm{Ab}$ & $30,74 \mathrm{Bb}$ & $12,33 \mathrm{Cb}$ & 0,0 & 0,0 & $39,92 b$ \\
\hline Água & $66,48 \mathrm{Ba}$ & $71,40 \mathrm{Aa}$ & $48,05 \mathrm{Ca}$ & $25,84 \mathrm{Da}$ & 0,0 & 0,0 & $59,94 \mathrm{a}$ \\
\hline $\mathrm{KNO}_{3} 0,1 \mathrm{M}$ & $65,08 \mathrm{Aa}$ & $48,34 \mathrm{Bb}$ & $33,74 \mathrm{Cb}$ & $18,21 \mathrm{Dab}$ & 0,0 & 0,0 & $41,34 \mathrm{~b}$ \\
\hline $\mathrm{KNO}_{3} 0,2 \mathrm{M}$ & $56,02 \mathrm{Aa}$ & $15,23 \mathrm{Ac}$ & $45,84 \mathrm{Aa}$ & $16,21 \mathrm{Aab}$ & 0,0 & 0,0 & $33,25 \mathrm{~b}$ \\
\hline \multirow[t]{2}{*}{ Média } & $61,47 \mathrm{~A}$ & $48,31 \mathrm{~B}$ & $39,59 \mathrm{~B}$ & $18,11 \mathrm{~B}$ & 0,0 & 0,0 & \\
\hline & \multicolumn{7}{|c|}{ Velocidade de germinação de sementes com punção do tegumento } \\
\hline Controle & 0,038 & 0,026 & 0,028 & 0,024 & 0,0 & 0,0 & $0,030 \mathrm{~b}$ \\
\hline Água & 0,051 & 0,042 & 0,034 & 0,032 & 0,0 & 0,0 & $0,040 \mathrm{a}$ \\
\hline $\mathrm{KNO}_{3} 0,1 \mathrm{M}$ & 0,048 & 0,031 & 0,031 & 0,037 & 0,0 & 0,0 & $0,034 \mathrm{ab}$ \\
\hline $\mathrm{KNO}_{3} 0,2 \mathrm{M}$ & 0,042 & 0,029 & 0,028 & 0,029 & 0,0 & 0,0 & $0,038 \mathrm{ab}$ \\
\hline \multirow[t]{2}{*}{ Média } & $0,044 \mathrm{~A}$ & $0,032 \mathrm{~B}$ & $0,030 \mathrm{~B}$ & $0,029 \mathrm{~B}$ & 0,0 & 0,0 & \\
\hline & \multicolumn{7}{|c|}{ Germinação de sementes sem punção do tegumento } \\
\hline Controle & $57,56 \mathrm{Ab}$ & $62,04 \mathrm{Ab}$ & $47,89 \mathrm{Ab}$ & $29,99 \mathrm{Bb}$ & $18,29 \mathrm{Ba}$ & $18,34 \mathrm{Ba}$ & $39,02 b$ \\
\hline Água & $68,43 \mathrm{Aa}$ & $87,11 \mathrm{Aa}$ & $62,58 \mathrm{Ba}$ & $43,84 \mathrm{Ca}$ & $27,25 \mathrm{Da}$ & $24,70 \mathrm{Da}$ & $52,32 \mathrm{a}$ \\
\hline $\mathrm{KNO}_{3} 0,1 \mathrm{M}$ & $65,80 \mathrm{Aa}$ & $74,93 \mathrm{Aab}$ & $45,38 \mathrm{Bb}$ & $30,74 \mathrm{Bab}$ & $24,83 \mathrm{Ca}$ & $11,53 \mathrm{Ca}$ & $42,20 \mathrm{ab}$ \\
\hline $\mathrm{KNO}_{3} 0,2 \mathrm{M}$ & $64,48 \mathrm{Aa}$ & $65,96 \mathrm{Aab}$ & $49,03 \mathrm{Bab}$ & $37,29 \mathrm{Bab}$ & $25,43 \mathrm{Ca}$ & $21,99 \mathrm{Ca}$ & $43,86 \mathrm{ab}$ \\
\hline \multirow[t]{2}{*}{ Média } & $63,82 \mathrm{~A}$ & $72,51 \mathrm{~A}$ & $51,22 \mathrm{~B}$ & $35,47 \mathrm{C}$ & $23,95 \mathrm{C}$ & $19,14 \mathrm{D}$ & \\
\hline & \multicolumn{7}{|c|}{ Velocidade de germinação de sementes sem punção do tegumento } \\
\hline Controle & 0,056 & 0,058 & 0,048 & 0,035 & 0,032 & 0,029 & $0,043 b$ \\
\hline Água & 0,075 & 0,075 & 0,056 & 0,042 & 0,038 & 0,029 & $0,052 \mathrm{a}$ \\
\hline $\mathrm{KNO}_{3} 0,1 \mathrm{M}$ & 0,060 & 0,070 & 0,056 & 0,044 & 0,029 & 0,031 & $0,049 b$ \\
\hline $\mathrm{KNO}_{3} 0,2 \mathrm{M}$ & 0,059 & 0,068 & 0,053 & 0,048 & 0,027 & 0,027 & $0,048 \mathrm{~b}$ \\
\hline Média & $0,062 \mathrm{~A}$ & $0,067 \mathrm{~A}$ & $0,053 \mathrm{AB}$ & $0,042 \mathrm{~B}$ & $0,032 \mathrm{~B}$ & $0,032 \mathrm{~B}$ & \\
\hline
\end{tabular}


ra e do tempo de exposição, os maiores valores de porcentagem foram obtidos em sementes condicionadas. A partir de 48 horas sob $50^{\circ} \mathrm{C}$, houve redução da viabilidade e do vigor das sementes, comprovados pela diminuição da porcentagem de germinação. Redução significativa da velocidade só foi registrada após 72 horas sob $50^{\circ} \mathrm{C}$. Sementes que permaneceram expostas a $60^{\circ} \mathrm{C}$ durante 72 horas perderam a viabilidade (Tabela 2).

As sementes que receberam a punção do tegumento e que foram condicionadas em água ou $\mathrm{KNO}_{3}$ apresentaram os maiores valores de porcentagem de germinação e diminuição significativa destes valores foi obtida a partir de 48 horas sob $50^{\circ} \mathrm{C}$. Com relação à velocidade, os valores médios mais elevados foram alcançados em sementes condicionadas em água e $\mathrm{KNO}_{3} 0,1 \mathrm{M}$. Decréscimos significativos deste parâmetro foram observados com a exposição a $50^{\circ} \mathrm{C}$ durante 72 horas (Tabela 2).

O estresse induzido por altas temperaturas pode levar à perda de habilidade de germinar em temperaturas ótimas. Proteínas desempenham um papel fundamental na modulação da resposta à temperatura, uma vez que altas temperaturas inativam enzimas, alteram a conformação dos lipídeos e desestruturam proteínas, pelas alterações nas conformações de peptídeos ou pela desestruturação de complexos protéicos na membrana celular (Jansen \& Ison, 1994). Desse modo, o uso do condicionamento melhora a qualidade das sementes, sob condições ideais para a germinação ou sob estresse hídrico, salino ou térmico (McDonald, 2000).

As sementes que não receberam o condicionamento apresentaram os maiores valores de condutividade e os menores valores foram obtidos em sementes condicionadas em água ou $\mathrm{KNO}_{3}$ 0,1 M. As sementes do grupo controle apresentaram os maiores valores de condutividade, e, portanto, menor vigor. Nas sementes com punção do tegumento, em comparação com as intactas, os valores de condutividade registrados foram dobrados, em todos os tipos de condicionamento empregados. Entre as sementes que receberam a punção, as de maior vigor foram aquelas condicionadas em água (Tabela 3).

O teste da condutividade elétrica é tido como um dos testes de vigor mais promissores quanto à possibilidade de padronização da metodologia, pelo menos conside-

Tabela 2. Germinação [arc sen (P/100) $)^{0,5}$ ] e velocidade de germinação (dias ${ }^{-1}$ ) de sementes de Chorisia speciosa armazenadas durante três anos, a $5^{\circ} \mathrm{C}$, submetidas ao condicionamento, com ou sem punção do tegumento, e, em seguida, expostas a estresse térmico em diferentes períodos.

\begin{tabular}{|c|c|c|c|c|c|c|c|c|}
\hline Condicionamento & Sem estresse & $50^{\circ} \mathrm{C}(24 \mathrm{~h})$ & $50^{\circ} \mathrm{C}(48 \mathrm{~h})$ & $50^{\circ} \mathrm{C}(72 \mathrm{~h})$ & $60^{\circ} \mathrm{C}(24 \mathrm{~h})$ & $60^{\circ} \mathrm{C}(24 \mathrm{~h})$ & $60^{\circ} \mathrm{C}(72 \mathrm{~h})$ & Média \\
\hline & \multicolumn{8}{|c|}{ Germinação de sementes com punção do tegumento } \\
\hline Controle & 52,39 & 41,20 & 38,50 & 35,40 & 34,40 & 28,84 & 0,0 & $38,45 \mathrm{ab}$ \\
\hline Água & 66,49 & 60,00 & 53,20 & 47,20 & 42,09 & 27,02 & 0,0 & $49,33 \mathrm{a}$ \\
\hline $\mathrm{KNO}_{3} 0,1 \mathrm{M}$ & 62,04 & 58,80 & 50,90 & 43,50 & 23,14 & 10,04 & 0,0 & $34,05 b$ \\
\hline $\mathrm{KNO}_{3} 0,2 \mathrm{M}$ & 59,02 & 55,00 & 49,67 & 34,60 & 29,17 & 8,00 & 0,0 & $33,36 \mathrm{~b}$ \\
\hline \multirow[t]{2}{*}{ Média } & $59,88 \mathrm{~A}$ & $53,75 \mathrm{AB}$ & $48,05 \mathrm{~B}$ & $40,18 \mathrm{BC}$ & $32,20 \mathrm{C}$ & $17,96 \mathrm{D}$ & 0,0 & \\
\hline & \multicolumn{8}{|c|}{ Velocidade de germinação de sementes com punção do tegumento } \\
\hline Controle & 0,143 & 0,129 & 0,114 & 0,114 & 0,104 & 0,090 & 0,0 & $0,106 b$ \\
\hline Água & 0,152 & 0,172 & 0,148 & 0,128 & 0,128 & 0,114 & 0,0 & $0,1485 \mathrm{a}$ \\
\hline $\mathrm{KNO}_{3} 0,1 \mathrm{M}$ & 0,157 & 0,176 & 0,117 & 0,117 & 0,106 & 0,100 & 0,0 & $0,113 \mathrm{ab}$ \\
\hline $\mathrm{KNO}_{3} 0,2 \mathrm{M}$ & 0,141 & 0,155 & 0,103 & 0,103 & 0,106 & 0,101 & 0,0 & $0,099 \mathrm{~b}$ \\
\hline \multirow[t]{2}{*}{ Média } & $0,146 \mathrm{~A}$ & $0,155 \mathrm{~A}$ & $0,134 \mathrm{~A}$ & $0,120 \mathrm{~B}$ & $0,111 \mathrm{~B}$ & $0,101 \mathrm{~B}$ & $0,0 \mathrm{C}$ & \\
\hline & \multicolumn{8}{|c|}{ Germinação de sementes sem punção do tegumento } \\
\hline Controle & 55,00 & 58,30 & 50,00 & 47,00 & 37,00 & 30,00 & 16,00 & $41,14 b$ \\
\hline Água & 88,00 & 79,00 & 72,00 & 54,00 & 48,00 & 31,00 & 30,00 & $57,42 \mathrm{a}$ \\
\hline $\mathrm{KNO}_{3} 0,1 \mathrm{M}$ & 80,00 & 62,04 & 58,00 & 57,00 & 33,14 & 28,50 & 28,00 & $49,25 \mathrm{ab}$ \\
\hline $\mathrm{KNO}_{3} 0,2 \mathrm{M}$ & 70,00 & 59,03 & 43,03 & 37,03 & 29,17 & 31,40 & 16,00 & $40,62 b$ \\
\hline \multirow[t]{2}{*}{ Média } & $73,25 \mathrm{~A}$ & $64,59 \mathrm{~A}$ & $55,75 \mathrm{~B}$ & $48,75 \mathrm{BC}$ & $36,82 \mathrm{CD}$ & $29,97 \mathrm{D}$ & $22,50 \mathrm{D}$ & \\
\hline & \multicolumn{8}{|c|}{ Velocidade de germinação de sementes sem punção do tegumento } \\
\hline Controle & 0,243 & 0,256 & 0,139 & 0,133 & 0,119 & 0,113 & 0,119 & $0,161 \mathrm{~b}$ \\
\hline Água & 0,236 & 0,380 & 0,330 & 0,229 & 0,192 & 0,154 & 0,178 & $0,243 \mathrm{a}$ \\
\hline $\mathrm{KNO}_{3} 0,1 \mathrm{M}$ & 0,273 & 0,306 & 0,210 & 0,224 & 0,125 & 0,135 & 0,090 & $0,194 b$ \\
\hline $\mathrm{KNO}_{3} 0,2 \mathrm{M}$ & 0,254 & 0,255 & 0,135 & 0,133 & 0,126 & 0,137 & 0,100 & $0,162 \mathrm{~b}$ \\
\hline Média & $0,252 \mathrm{~A}$ & $0,222 \mathrm{~A}$ & $0,203 \mathrm{~A}$ & $0,125 \mathrm{~B}$ & $0,139 \mathrm{~B}$ & $0,136 \mathrm{~B}$ & $0,139 \mathrm{~B}$ & \\
\hline
\end{tabular}

${ }^{(1)}$ Médias seguidas pelas mesmas letras, maiúsculas nas linhas e minúsculas nas colunas, não diferem entre si pelo teste de Tukey a 5\% de probabilidade; os coeficientes de variação para a germinabilidade e velocidade de germinação são, respectivamente, 18,3\% e 19,40\%, nas sementes com punção do tegumento, e 12,05\% e 18,10\%, em sementes sem punção. 
Tabela 3. Valores médios de condutividade elétrica $\left(\mathrm{mS} \mathrm{cm}^{-1} \mathrm{~g}^{-1}\right)$ de sementes de Chorisia speciosa armazenadas durante três anos, a $5^{\circ} \mathrm{C}$, com ou sem punção do tegumento, e condicionadas a $27^{\circ} \mathrm{C}$, durante 24 horas em água e $\mathrm{KNO}_{3}$ a $0,1 \mathrm{M}$ e $0,2 \mathrm{M}^{(1)}$.

\begin{tabular}{lcc}
\hline Condicionamento & Sem punção & Com punção \\
\hline Sem condicionamento & $156,0 \mathrm{a}$ & $282 \mathrm{a}$ \\
Água & $91,4 \mathrm{c}$ & $195 \mathrm{~b}$ \\
$\mathrm{KNO}_{3} 0,1 \mathrm{M}$ & $103,4 \mathrm{bc}$ & $234 \mathrm{a}$ \\
$\mathrm{KNO}_{3} 0,2 \mathrm{M}$ & $106,5 \mathrm{~b}$ & $261 \mathrm{a}$ \\
\hline
\end{tabular}

${ }^{(1)}$ Médias seguidas pelas mesmas letras, em uma mesma coluna, não diferem entre si pelo teste de Tukey a 5\% de probablidade.

rando uma mesma espécie (Association of Official Seed Analyst, 1983). A partir do teste de condutividade elétrica é possível quantificar com rapidez, precisão e eficácia, a qualidade das sementes por meio da avaliação das transformações degenerativas verificadas em suas membranas celulares e tegumentos (Sampaio et al., 1995).

\section{Conclusões}

1. O limite de tolerância ao estresse salino não é reduzido após três anos de armazenamento.

2. As sementes de paineira condicionadas em água apresentam maior vigor e viabilidade sob estresse salino.

3. A sensibilidade ao estresse térmico diminui após o armazenamento e o condicionamento não reverte este efeito.

4. O condicionamento pode substituir o pré-tratamento punção do tegumento para uniformizar e acelerar o processo germinativo.

5. A punção do tegumento não é indicada para sementes armazenadas.

\section{Agradecimento}

Ao CNPq, pelo apoio financeiro.

\section{Referências}

AGBOOLA, D.A. Effect of saline solutions and salt stress on seed germination of one tropical forest tree species. Revista de Biologia Tropical, v.46, p.1109-1115, 1998.

ASSOCIATION OF OFFICIAL SEED ANALYST. Seed vigor testing handbook. East Lansing: AOSA, 1983. 88p. (Contribution, 32).
BRACCINI, A.L.; REIS, M.S.; SEDIYAMA, C.S.; SCAPIM, C.A.; BRACCINI, M.C.L.M. A avaliação da qualidade fisiológica de sementes de soja após o processo de hidratação-desidratação e envelhecimento acelerado. Pesquisa Agropecuária Brasileira, v.34, p.1053-1066, 1999.

CARPI, S.M.F.; BARBEDO, C.J.; MARCOS FILHO, J. Condicionamento osmótico de sementes de Cedrela fissilis Vell. Revista Brasileira de Sementes, v.18, p.271-275, 1996.

CARVALHO, P.R. Espécies florestais brasileiras: recomendações silviculturais, potencialidade e uso de madeira. Colombo, PR: Embrapa-CNPF, 1994. 640p.

CASTRO, R.D.C.; HILHORST, H.W.M. Embebição e reativação do metabolismo. In: FERREIRA, A.G.; BORGHETTI, F. Germinação: do básico ao aplicado. Porto Alegre: Artmed, 2004. p.149-162.

FANTI, S.C. Aspectos da germinação e efeitos do condicionamento osmótico em sementes de paineira (Chorisia speciosa St. Hil - Bombacaeae). 2001. 145p. Tese (Doutorado) Universidade Federal de São Carlos, São Carlos.

FANTI, S.C.; PEREZ, S.C.J.G. de A. Efeitos do estresse hídrico e do envelhecimento precoce na viabilidade de sementes osmocondicionadas de sementes de paineira (Chorisia speciosa). Pesquisa Agropecuária Brasileira, v.38, p.537-544, 2003.

GRAPHPAD InSTAT, Software. The Instat guide to choosing and interpreting statistical tests: a manual for GraphPad InStat. Version 3.0. San Diego: Oxford University Pres, 1999. 123p.

JANSEN, P.I.; ISON, R.L. Temperature effects on germination of Trifolium balenssae and $T$. resupinatum with special reference to hight temperature dormancy. Australian Journal of Agricultural Research, v.45, p.689-701, 1994.

JELLER, H.; PEREZ, S.C.J.G. de A. Condicionamento osmótico na germinação de sementes de cássia do nordeste sob estresse hídrico, térmico e salino. Pesquisa Agropecuária Brasileira, v.38, p.10251034, 2003.

LABOURIAU, L.G. A germinação de sementes. Washington: Secretaria da O.E.A, 1983. 173p.

McDONALD, M.B. Seed priming. In: BLACK, M.; BEWLEY, J.D. Seed technology and its biological basis. Sheffield: Sheffield Academic Press, 2000. p.287-325.

MUHYADDIN, T.; WIEBE, H.J. Effects of seed treatments with polyethylene glicol (PEG) on emergence of vegetable crops. Seed Science and Technology, v.17, p.49-56, 1989.

PEREZ, S.C.J.G. de A.; NEGREIROS, G.F. Efeitos do précondicionamento na viabilidade e no vigor de sementes de canafístula (Peltophorum dubium (Spreng) Taub.) em condições de estresse. Revista Brasileira de Sementes, v.23, 175-183, 2001.

PINÃ-RODRIGUES, F.C.M.; JESUS, R.M. Comportamento das sementes de cedro rosa (Cedrela angustifolia) durante o armazenamento. Revista Brasileira de Sementes, v.14, p.31-36, 1992.

SALISBURY, F.B.; ROSS, C.W. Plant physiology. 4.ed. California: Wadsworth Publishing Company, 1994. 682p.

SAMPAIO, N.V.; GIMENEZ-SAMPAIO, T.; DURAN, J.M. Estudos de variáveis na leitura de condutividade elétrica com um 
analisador automático de sementes modelo ASAC-100. Revista Brasileira de Sementes, v.17, p.197-204, 1995.

SUÑE, A.D.; FRANKE, L.B.; SAMPAIO, T.G. Efeitos do condicionamento osmótico na qualidade fisiológica de sementes de Adesmia latifolia (Spreng.) Vog. Revista Brasileira de Sementes, v.24, p.18-23, 2002.

TAIZ, L.; ZEIGER, E. Fisiologia vegetal. 3.ed. Trad. Eliane Romanto Santarém. Porto Alegre: Artmed, 2004. 719p.

TAYLOR, A.G.; ALLEN, P.S.; BENNETT, M.A.; BRADFORD, K.J.; BURRIS, J.S.; MISRA, M.K. Seed enhancements. Seed Science Research, v.8, p.245-256, 1998.
VASQUEZ, G.H. Condicionamento fisiológico de sementes de soja: efeitos sobre a germinação, vigor e potencial de armazenamento. 1995. 138p. Dissertação (Mestrado) - Escola Superior de Agricultura Luiz de Queiroz, Piracicaba.

WANLI, Z.; LEIHONG, L.; PEREZ, S.C.J.G. de A. Efeitos do précondicionamento em sementes de canafístula (Peltophorum dubium (Spreng) Taub.). Revista Brasileira de Sementes, v.23, p.146156, 2001.

WARREN, J.E.; BENETT, M.A. Seed hydration using the drum priming system. HortScience, v.32, p.1220-1221, 1997.

ZAR, J.H. Biostatistical analysis. $4^{\text {th }}$ ed. Upper Saddle River: Prentice Hall, 1999. 663p.

Recebido em 24 de março de 2004 e aprovado em 24 de novembro de 2004 